\title{
The use of transformed IMR90 cell model to identify the potential extra-telomeric effects of $h T E R T$ in cell migration and DNA damage response
}

\author{
Xu Cao ${ }^{1}$, Chiou Mee Kong ${ }^{1}$, Kanchi Madhu Mathi ${ }^{1}$, Yoon Pin Lim', Valere Cacheux-Rataboul ${ }^{2}$ \\ and Xueying Wang ${ }^{1,3^{*}}$
}

\begin{abstract}
Background: Human telomerase reverse transcriptase (hTERT), the catalytic subunit of telomesase, is responsible for telomere maintenance and its reactivation is implicated in almost 90\% human cancers. Recent evidences show that hTERT is essential for neoplastic transformation independent of its canonical function. However, the roles of hTERT in the process remain elusive. In the current work, we explore the extra-telomeric role of hTERT in the neoplastic transformation of fibroblast IMR90.

Results: Here we established transformed IMR90 cells by co-expression of three oncogenic factors, namely, H-Ras, SV40 Large-T antigen and hTERT (RSH). The RSH-transformed cells acquired hallmarks of cancer, such as they can grow under anchorage independent conditions; self-sufficient in growth signals; attenuated response to apoptosis; and possessed recurrent chromosomal abnormalities. Furthermore, the RSH-transformed cells showed enhanced migration capability which was also observed in IMR90 cells expressing hTERT alone, indicating that hTERT plays a role in cell migration, and thus possibly contribute to their metastatic potential during tumor transformation. This notion was further supported by our microarray analysis. In addition, we found that Ku70 were exclusively upregulated in both RSH-transformed IMR90 cells and hTERT-overexpressing IMR90 cells, suggesting the potential role of hTERT in DNA damage response (DDR).
\end{abstract}

Conclusions: Collectively, our study revealed the extra-telomeric effects of hTERT in cell migration and DDR during neoplastic transformation.

Keywords: Extra-telomeric, hTERT, Neoplastic transformation, Cell migration, DNA damage response

\section{Background}

Human telomeres are TTAGGG repeats at the ends of human chromosomes, protecting them from end-to-end fusion and maintaining chromosomal stability $[1,2]$. In human somatic cells, telomere length shortens in each cell division as DNA polymerase is unable to replicate the very end of telomere [3,4]. Eventually cells stop

\footnotetext{
* Correspondence: bchwxy@nus.edu.sg

'Department of Biochemistry, Yong Loo Lin School of Medicine, National University of Singapore, Block MD4, Level 1, 5 Science Drive 2, Singapore 117545, Singapore

${ }^{3}$ National University Cancer Institute of Singapore (NCIS), Singapore, Singapore

Full list of author information is available at the end of the article
}

proliferating and undergo senescence. However, almost $90 \%$ of human cancers overcome the finite divisional potential by reactivation of a ribonucleoprotein complex, known as telomerase [5-7]. The catalytic subunit of telomerase, telomerase reverse transcriptase (hTERT), is the rate-limiting factor of telomerase activity. Upregulation of hTERT confers cells limitless proliferative potential, which is one of the cancer hallmarks [8].

Emerging evidence suggests that maintenance of telomere length might not the sole function of hTERT during oncogenesis. For instance, knockdown of hTERT resulted in rapid inhibition of cell proliferation and growth in cancer cells without affecting the telomere length [9]. 
Moreover, overexpressing hTERT in mice with long telomeres attributed to increase risk of cancer formation [10], which further support the notion that hTERT has other functions, on top of its canonical function in telomere maintenance. In addition, human cells utilize alternative lengthening of telomeres pathway for telomere maintenance can only be transformed when co-expressing hTERT and oncogenic-Ras, indicating that hTERT is indispensable for cancer transformation [11,12]. Taken together, these findings suggest that hTERT plays a key role in tumorigenesis independent of its canonical function. Its roles in neoplastic transformation, however, are still not well understood. Therefore, investigating the roles of hTERT in the cancer transformation is of utmost imperative.

Neoplastic transformation can be achieved by in vitro genetic manipulation. Studies showed that disruption of the intracellular pathways regulated by SV40 Large-T, oncogenic Ras and hTERT are sufficient to create a human tumor cell [13]. This highlighted the various pathways that require changes for transformation to occur: the mitogenic response pathway activated by Ras [14]; telomere maintenance pathway by hTERT [4]; cell surveillance pathways due to the functional abolishment of p53 and Rb tumor-suppressors by Large-T [15]. Since disruption of these cellular pathways are commonly seen in in vivo tumors, tumor cells generated from such transformed cell model can be a good representation of actual human cancers [16]. This model also serves as a platform to study the early stages of the tumor formation, as compared to tumor biopsies that are often obtained at an advanced stage [13].

Here, we transformed IMR90, a non-epithelial somatic lung fibroblast, by three factors, including H-Ras, SV40 Large-T, and hTERT (RSH). Using the RSH-transformed IMR90 cell model, our results unveiled the extratelomeric functions of hTERT in cell migration as well as in DNA damage response during neoplastic transformation. Therefore, our findings suggest that hTERT is an attractive target for cancer therapy, even at early stage of cancer formation.

\section{Results and discussion}

RSH-transformed cells acquire cancer cells characteristics Primary human fibroblast cells IMR90 were successfully co-transfected with Ras, SV40 Large-T, and hTERT and their protein expressions were confirmed by western blotting (Figure 1A). Morphologically, IMR90 RSH fibroblasts appeared to be shorter and rounder compared to the infection control (Figure 1B). This observation is consistent with the findings of Mason and colleagues in IMR90 cells transformed with E1a/Ras [17], suggesting that these changes are the unique characteristics of cellular transformation. Moreover, late passages of IMR90 control cells underwent significant increase in cell sizes, indicating their senescent status. However, this was not observed in IMR90 RSH cells even after several passages (data not shown).

One of the hallmarks of the cancerous cells is they can survive and grow in the absence of anchorage to the extracellular matrix [18]. Our anchorage independent growth assays demonstrated that IMR90 RSH cells formed small microscopic colonies ( $<200 \mu \mathrm{m}$ in diameter) while MCF-7 cells, the positive control, formed large visible colonies (>200 $\mu \mathrm{m}$ in diameter) (Figure 1C) after 6 weeks. Comparison of colony sizes with MCF-7 suggests that transformation by three genetic factors produced cells that were less tumorigenic than the established cancer cells. Thus, the RSH-transformed cell could serve as a representative model to study the early events of cancer transformation, compared to an established cell line.

Another common trait of cancer cells is their selfsufficiency in growth signals [18]. To investigate the effects of growth factors withdrawal on the RSH-transformed cells, the cells were subjected to a serum-free environment, following which cell proliferation and survival were assessed over a three-day period. As expected, IMR90 control cells showed signs of apoptosis after 24 hours of serum withdrawal. However, no apoptosis was observed in IMR90 RSH cells even after treating for 72 hours in serum-free condition, suggesting that these RSH-transformed cells were able to survive in the absence of growth factors (Figure 1D). We further tested whether transformed fibroblasts are refractory to the induction of apoptosis, which is commonly observed in cancer cells [19]. After treating with Doxorubicin (Dox), a DNA damageinducing drug for 48 hours, IMR90 RSH cells were able to survive even at much higher Dox concentrations $(3 \mu \mathrm{M}$ and $5 \mu \mathrm{M}$ ) than IMR90 control cells, reflecting an attenuation in the apoptotic machinery of the cells (Figure 1E).

For potential use of IMR90 RSH as a cancer cell model, we also assessed their chromosomal aberrations for recurrent abnormalities through cytogenetics study [20]. Karyotyping of IMR90 RSH cells revealed recurrent abnormalities (Figure 1F,G). Moreover, spectral karyotyping analysis revealed that $60.9 \%$ (14 out of 23) of IMR90 RSH cells had recurrent chromosomal abnormalities at chromosomes 4, 18, 20 (Figure 1H; Additional file 1: Table S1). Taken together, these data indicated that IMR90 cells were successfully transformed by co-expressing three oncogenic factors. The RSH-transformed fibroblasts acquired various characteristics of a human cancer cell and may serve as a valuable model to study the early events of tumorigenesis.

\section{RSH-transformed cells and hTERT-overexpressing cells demonstrate increased migration capability}

Metastasis is often correlated to two attributes: migration followed by invasion [21]. Metastatic tumors are believed to occur in the late stages of cancer [22]. However, in 


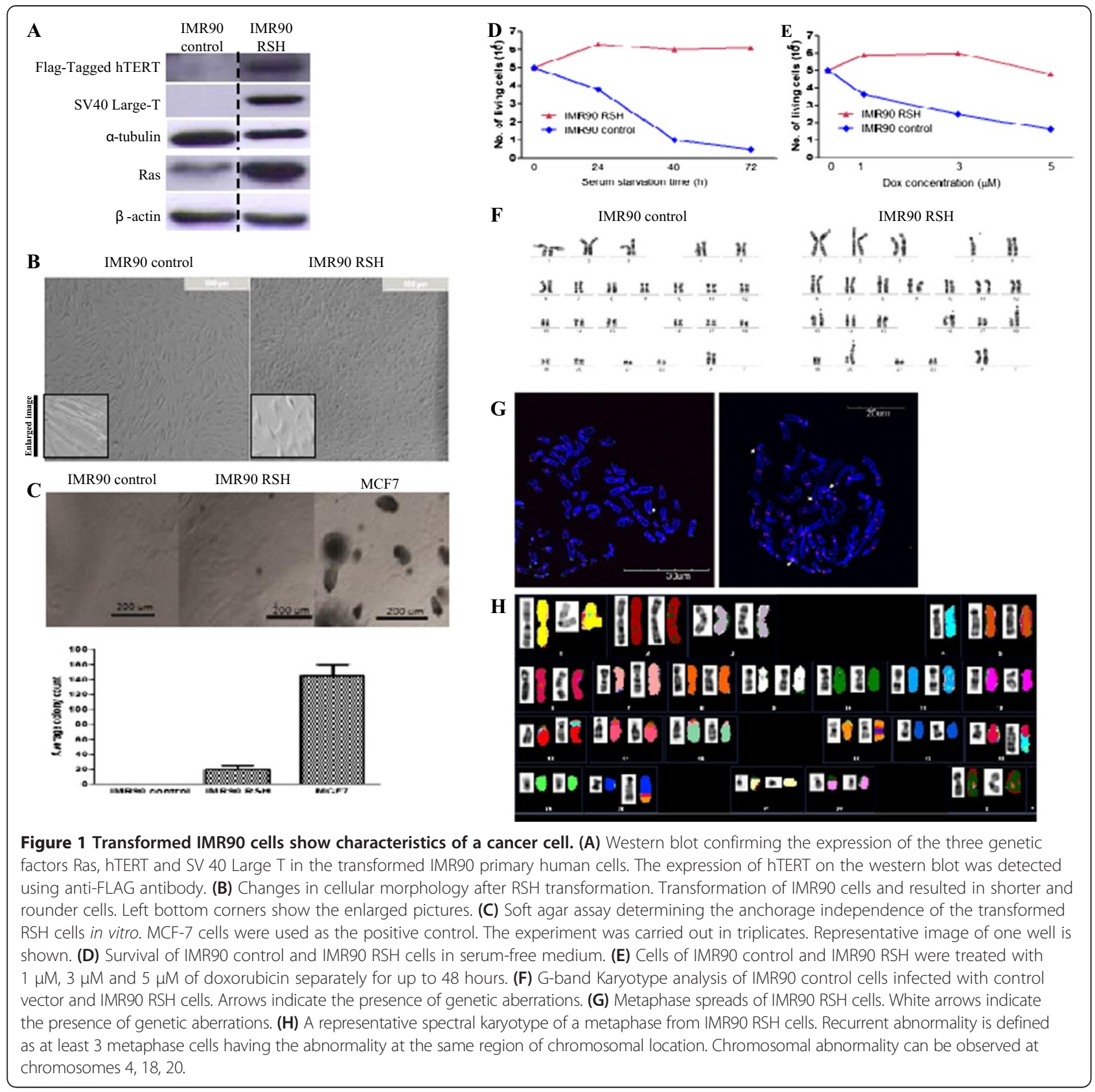

numerous early-stage cancers, recurrence can be observed even after the removal of non-invasive, benign tumors, suggesting the possibility of the cancer cells undergoing metastasis at a much earlier stage [23]. We questioned if IMR90 RSH cells possess migration capability similar to that of a cancer cell. Our Boyden assay results showed that IMR90 RSH cells had a greater migration capability as compared to IMR90 control cells (Figure 2A). This result was further validated with wound healing assay, in which the gap was reduced to $40 \%$ for IMR90 RSH cells compared to $84 \%$ in IMR90 control cells $(p<0.01)$ (Figure $2 \mathrm{~B}, \mathrm{C})$. Moreover, we observed that IMR90 RSH cells migrated faster and in a more individualistic pattern compared to IMR90 control cells, implying some degree of autonomy (Figure 2B).

Given that transformation can increase the migration capability of cells and that hTERT is one of the upregulated factors in the transformed cells, it then raised the question as to whether hTERT alone can contribute to this phenomenon. In order to assess the possible role of telomerase in cell migration, we also performed wound healing assay on IMR90 cells expressing hTERT alone. Similar to IMR90 RSH cells, the hTERT-overexpressing IMR90 cells (Figure 2D) also migrated faster than IMR90 control cells (Figure 2E). However, when compared to IMR90 


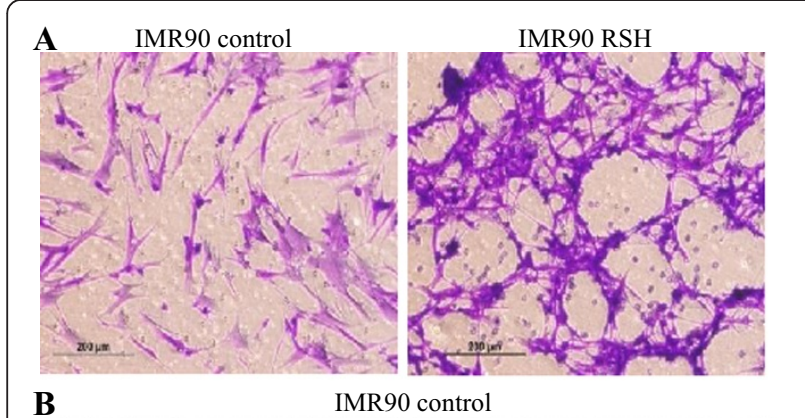

B

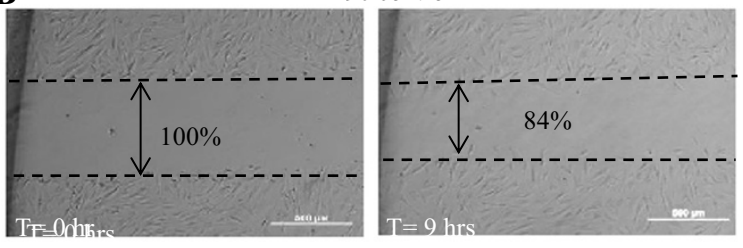

IMR90 RSH
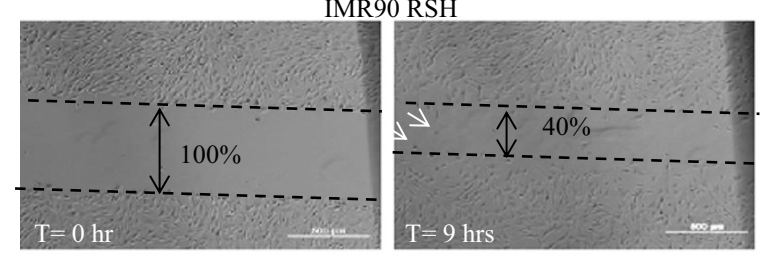

C

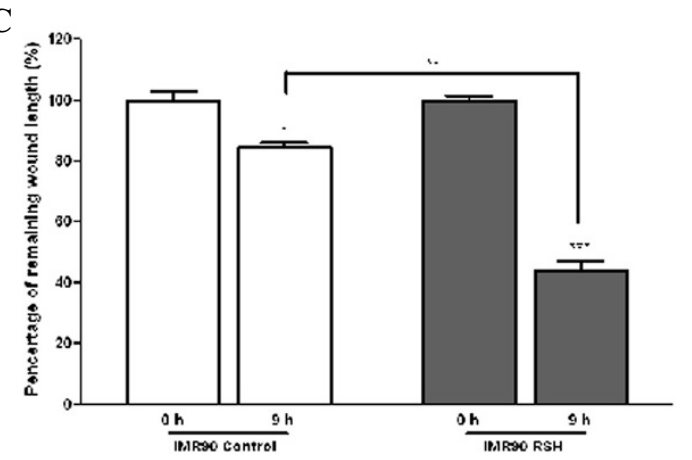

D

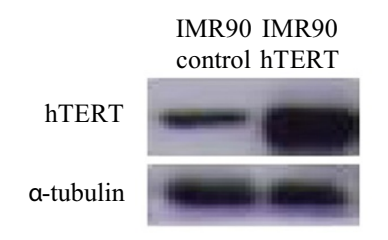

E
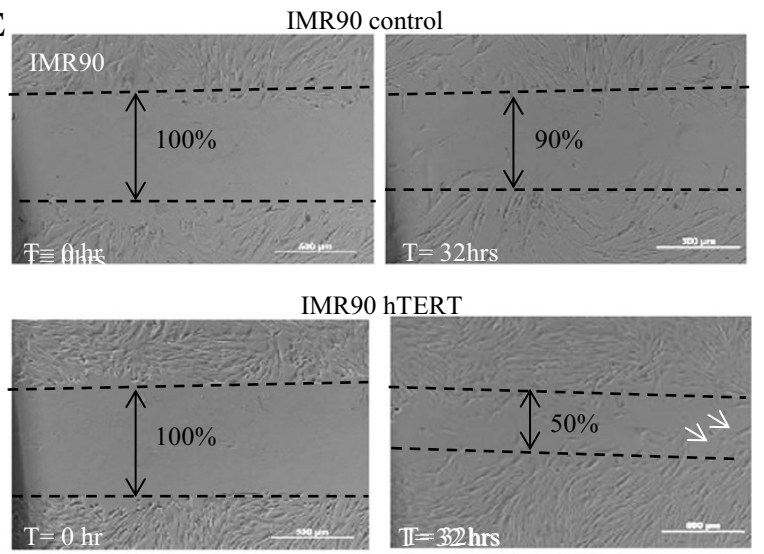

$\mathbf{F}$

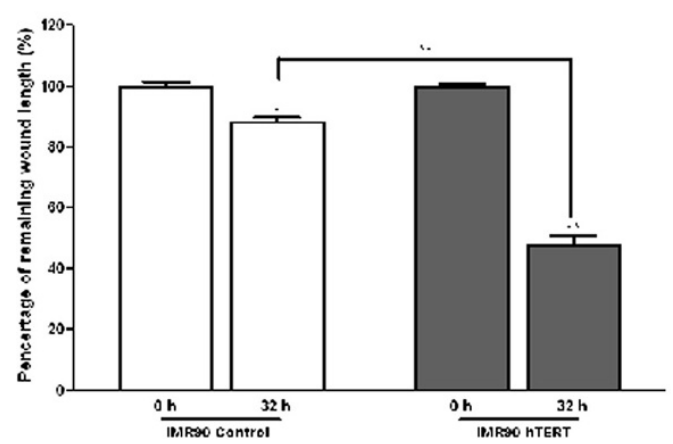

Figure 2 Migration capability analysis of IMR90 RSH and IMR90 hTERT cells. (A) Boyden assay comparing the migration capability of IMR90 control and IMR90 RSH cells after 10 hours. (B) Wound healing assay comparing the migration of IMR90 control and IMR90 RSH cells after 9 hours of incubation. Images at 0 hour and at 9 hours, representative of triplicate experiments for IMR90 control and IMR90 RSH cells, are shown. White arrows indicate individual cells that have migrated. (C) The 'wound closure' areas are visualized under an inverted microscope and bar graphs show the distance travelled by IMR90 control and IMR90 RSH cells in the wound healing assay. Results are indicated as the mean \pm standard deviation (SD) $(n=2) .{ }^{*} p<0.05 ;{ }^{* *} p<0.01 ;{ }^{* *} p<0.001$. (D) Western blot confirming the overexpression of hTERT in IMR90 primary human cells. (E) Wound healing assay comparing the migration of IMR90 control and IMR90 hTERT cells after 32 hours of incubation. Images at 0 hour and at 32 hours, representative of triplicate experiments for IMR90 control and IMR90 hTERT cells, are shown. White arrows indicate individual cells that have migrated. (F) Bar graphs showing the distance travelled by IMR90 control and IMR90 hTERT cells in the wound healing assay. Results are indicated as the mean \pm standard deviation (SD) $(n=2) .{ }^{*} p<0.05 ;{ }^{* *} p<0.01 ;{ }^{* *} p<0.001$.

RSH cells, the migration process in IMR90 hTERT cells started to occur only after 10 hours and they seemed to have a slower migration rate. As shown in Figure 2E and F, IMR90 hTERT cells migrated about 50\% distance, yet the distance was only reduced by $10 \%$ for IMR 90 control cells $(p<0.01)$. Collectively, these suggest that hTERT does play a role in cell migration, which in turn contributes to the metastatic potential of cancer cells.
Microarray analysis supports the notion that hTERT plays a role in cell migration

To investigate the underlying molecular basis of hTERTinduced migration in transformed cells, we analyzed the genome-wide gene expression in IMR90 RSH cells and IMR90 hTERT cells. There were a total of 62 and 150 migration-related genes that were found to be differentially expressed (fold change $\geq 2 ; p<0.05$ ) in IMR90 RSH 
cells and IMR90 hTERT cells respectively. Among these differentially expressed genes, 28 genes were found to be overlapped in both IMR90 RSH and IMR90 hTERT cells (Table 1), suggesting that these genes may attribute to migration during neoplastic transformation and specifically, they were under the regulation of hTERT. Approximately $93 \%$ of the genes were found to be up-regulated by hTERT. Remarkably, pro-inflammatory factors such as Interleukin-6 (IL6) and IL8 were significantly enhanced in both RSH-transformed cells and hTERT-overexpressing cells. Previous published data demonstrated that IL6 and IL8 are targets under hTERT-mediated regulation of NF- $\mathrm{kB}$ pathway [24]. On top of their role in inflammation, they are also found to be involved in tumor cell migration and invasion [25], which is corroborated with our microarray data. Taken together, it is reasonable to hypothesize that hTERT could enhance the cell migration through NF-kB pathway in early tumorigenesis.

On the other hand, Liu et al. in 2013 have shown that hTERT promotes the epithelial-mesenchymal transition (EMT) by upregulating snail family zinc finger 1 (Snail1) and vimentin through Wnt/beta-catenin signaling pathway in gastric cancer [26]. We did not observe significant changes in Snail-1 and vimentin genes expressions in our cell models and this could be attributed to different cell model used in both studies. However, we noticed that two downstream genes of Wnt/beta-catenin pathways, namely Cyclooxygenase-2 (COX-2) and Twist1 were significantly enhanced in both RSH-transformed cells and

Table 1 Changes in migration-related genes in both IMR90 RSH and IMR90 hTERT cells

\begin{tabular}{|c|c|c|c|c|}
\hline \multirow{2}{*}{$\begin{array}{c}\text { Gene } \\
\text { symbol }\end{array}$} & \multirow[t]{2}{*}{ Gene name } & \multirow[t]{2}{*}{ Function on cell migration } & \multicolumn{2}{|c|}{ Fold change } \\
\hline & & & IMR90 RSH vs control & IMR hTERT vs control \\
\hline IL6 & Interleukin 6 & Positive regulation of cell migration & 14.135 & 2.665 \\
\hline APOE & Apolipoprotein E & Negative regulation of cell motion & 7.82747 & 5.968 \\
\hline $\operatorname{cox}-2$ & Cyclooxygenase-2 & Positive regulation of cell migration & 6.094 & 2.475 \\
\hline LAMA5 & Laminin, alpha 5 & Positive regulation of cell migration & 4.273 & 2.498 \\
\hline IL8 & Interleukin 8 & Positive regulation of locomotion & 3.755 & 2.588 \\
\hline BTG1 & B-cell translocation gene 1 & Positive regulation of cell migration & 3.34 & 3.392 \\
\hline SCG2 & Secretogranin II & Positive regulation of locomotion & 3.051 & 3.563 \\
\hline ETV4 & Ets variant 4 & Positive regulation of cell migration & 3.047 & 2.884 \\
\hline CKLF & Chemokine-like factor & Positive regulation of cell migration & 2.865 & 3.246 \\
\hline COL18A1 & Collagen, type XVIII, alpha 1 & Positive regulation of cell migration & 2.738 & 4.533 \\
\hline LAMA1 & Laminin, alpha 1 & Positive regulation of cell migration & 2.631 & 6.232 \\
\hline LAMA4 & Laminin, alpha 4 & Positive regulation of cell migration & 2.582 & 2.3 \\
\hline MYH10 & Myosin, heavy chain 10 , non-muscle & Positive regulation of cell migration & 2.495 & 5.678 \\
\hline BBS2 & Bardet-Biedl syndrome 2 & Positive regulation of cell migration & 2.479 & 2.415 \\
\hline WASF2 & WAS protein family, member 2 & Positive regulation of cell migration & 2.421 & 5.506 \\
\hline TWIST1 & Twist homolog 1 (Drosophila) & Positive regulation of cell migration & 2.409 & 3.328 \\
\hline NR4A2 & Nuclear receptor subfamily 4 , A2 & Positive regulation of cell migration & 2.398 & 3.488 \\
\hline SMAD3 & SMAD family member 3 & Positive regulation of cell migration & 2.396 & 3.602 \\
\hline PLAT & Plasminogen activator, tissue & Positive regulation of cell migration & 2.367 & 3.977 \\
\hline SEMA3F & Semaphorin $3 \mathrm{~F}$ & Positive regulation of cell migration & 2.348 & 5.71 \\
\hline NUP85 & Nucleoporin 85 kDa & Positive regulation of cell migration & 2.214 & 3.579 \\
\hline PALM & Paralemmin & Positive regulation of cell migration & 2.151 & 2.42 \\
\hline $\mathrm{ROBO} 3$ & Roundabout, axon guidance receptor, homolog 3 & Positive regulation of cell migration & 2.116 & 2.929 \\
\hline NDE1 & nudE nuclear distribution gene E homolog 1 & Positive regulation of cell migration & 2.115 & 2.388 \\
\hline CDKN1B & Cyclin-dependent kinase inhibitor $1 \mathrm{~B}$ & Negative regulation of cell motion & 2.068 & 2.316 \\
\hline LYN & v-yes-1 Yamaguchi sarcoma oncogene & Positive regulation of cell motion & 2.032 & 4.017 \\
\hline PRKDC & Protein kinase, DNA-activated, catalytic polypeptide & Positive regulation of cell migration & 2.024 & 3.337 \\
\hline ACTG1 & Actin, gamma 1 & Positive regulation of cell migration & -5.041 & -3.107 \\
\hline
\end{tabular}


hTERT-overexpressing cells. Both were reported to be implicated in cancer cell migration [27,28], and this is in consistent with our microarray data. Taken together, our microarray data complements with our previous notion that hTERT play an important role in cell mobility, which in turn contributes to the metastatic potential of cancer cells.

\section{hTERT might be implicated in DNA damage response via upregulating Ku70 during IMR90 transformation}

To delineate the roles of hTERT in neoplastic transformation, we performed mass spectrometry analysis on the protein levels of IMR90 RSH cells. Surprisingly, analysis by mass spectrometry revealed that protein $\mathrm{Ku} 70$ was exclusively found in IMR90 RSH cells, but not in IMR90 control cells (Figure 3A; Additional file 2: Table S2). Moreover, both RT-PCR and immunoblotting results confirmed our observation where Ku70 expression was augmented in IMR90 RSH as well as IMR90 hTERT cells, but not in IMR90 control cells (Figure 3B,C). This could be indicative of an activated DNA damage responses (DDR) in both IMR90-RSH and IMR90-hTERT since Ku70 is a DDR sensor and is implicated in the non homologous end joining (NHEJ) pathway [29]. Ku70 forms a heterodimeric complex with Ku80 [30]. However, the protein expression of Ku80 remained unchanged (Figure 3C). Presence of Ku70 in IMR90 RSH cells and IMR90 hTERT cells prompted us to assess whether other DDR-associated proteins could be regulated by hTERT as well. Our microarray data revealed that several DDR-associated genes were upregulated in both RSH-transformed IMR90 and hTERToverexpressing IMR90 cells (fold change $\geq 2 ; p<0.05$ ) (Additional file 3: Table S3). Taken together, these results suggest that hTERT may play an important role in DDR pathways, via Ku70 and other DDR-associated proteins.

\section{Conclusions}

In conclusion, the neoplastic transformation of human primary fibroblast cells using SV40 Large-T antigen, $\mathrm{H}$-Ras and hTERT can be used as an in vitro cell model for cancer studies, especially for those fibroblasts originated tumors. Moreover, our findings suggest that hTERT is implicated in cell migration as well as DDR during neoplastic transformation. As these processes have great implications in cancer progression, our study could provide insights on the roles of hTERT and its underlying mechanisms in human cancer formation.

\section{Methods}

\section{Cell lines}

Human breast cancer cells MCF-7 (HTB-22) and normal human fibroblasts, including IMR90 (CCL-186) and BJ (CCL-186) were purchased from American Type Culture Collection (ATCC). Cells were cultured in Dulbecco's Modified Eagle's Medium (DMEM) supplemented with $10 \%$ fetal bovine serum (FBS) (Gibco, Invitrogen), $2 \mathrm{mM}$ L-glutamine (Gibco, Invitrogen) and 100U/ml penicillin and streptomycin (Gibco, Invitrogen).

\section{Infection and selection of cells}

Retroviral pBabe-puro Ras V12 (Addgene Plasmid 1768), and retroviral pBabe-puro SV40 LT (Addgene Plasmid 13970) were purchased from Addgene. FLAG was tagged to the N-terminus of hTERT and cloned into a lentiviral vector with Ires hygromycin mammalian selection. Retroviral pBabe-puro-hTERT vector (Addgene Plasmid 1771)
A

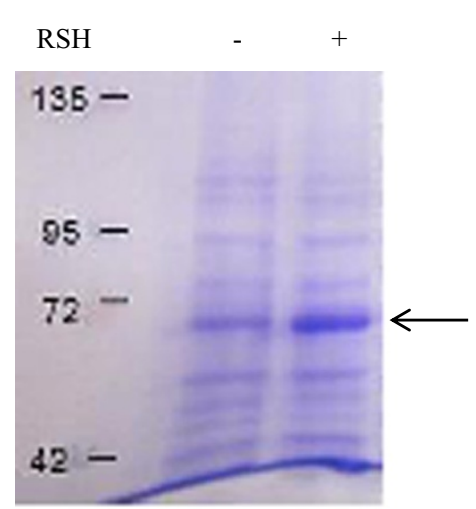

B

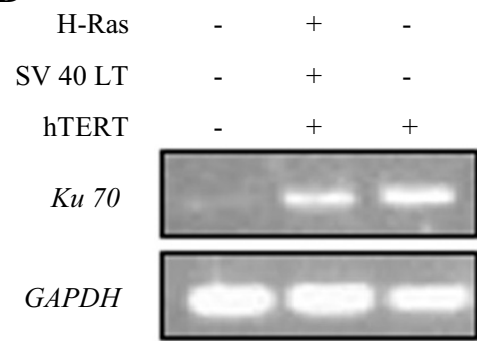

C

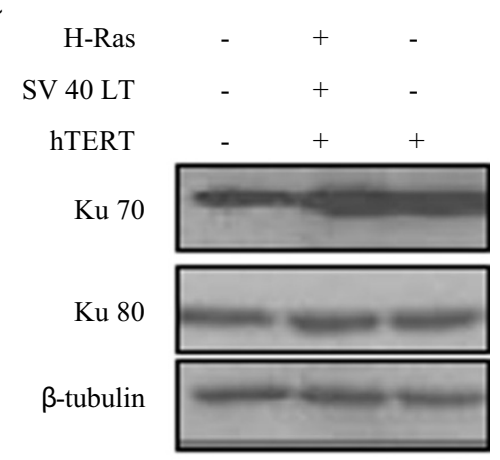

Figure 3 Ku 70 expression in IMR90-RSH and IMR90 hTERT cells. (A) Coomassie blue staining of the protein expression in RSH transformed cells and control fibroblasts, showing an augmented $70 \mathrm{kDa}$ (indicated by the black arrow). (B) Semi-quantitative analysis of Ku70 by RT-PCR in IMR90 control, IMR90 hTERT and IMR90 RSH cells. Ku70 expression showed an increase in IMR90 RSH and IMR90 hTERT cells compared to IMR90 control cells. (C) Ku70 protein expression in IMR90 control, IMR90 hTERT and IMR90 RSH cells. Ku70 protein expression showed an increase in IMR90 RSH and IMR90 hTERT cells compared to IMR90 control cells. 
was used to generate hTERT-overexpressing cell. Retroviral pBabe-puro vector (Addgene Plasmid 1764) was used as infection control. IMR90 was infected with virus supernatant with $8 \mu \mathrm{g} / \mathrm{ml}$ of polybrene for 24 hours. To generate stable cell line that co-expresses Ras, SV40 Large T and hTERT, cells infected with RSH combination were selected using both puromycin and hygromycin (Invitrogen) for 5 days. Cells infected with pBabe-puro-hTERT as well as pBabe-puro control vector were selected using puromycin (InvivoGen) for 5 days. Cells stably expressing the desired genes were further passaged and maintained on selection medium for an additional two to three weeks prior to downstream experiments.

\section{Anchorage-independent growth assay}

$10^{4}$ cells were seeded in $0.3 \%(\mathrm{w} / \mathrm{v})$ agarose with DMEM and $16.66 \%$ FBS onto each well of a 24-well plate with a $0.6 \%(\mathrm{w} / \mathrm{v})$ agarose underlay. Wells were analyzed for colony formation after 6 weeks. Scoring was done by counting the colonies under the microscope.

\section{Serum-free cell survival assay}

$10^{5}$ cells were seeded and the number of living cells was determined by Countess automated cell counter (Invitrogen) after treating them in serum-free DMEM for 24,48 and 72 hours.

\section{Drug treatment}

$10^{5}$ cells were treated with Doxorubicin (Calbiochem) at $1 \mu \mathrm{M}, 3 \mu \mathrm{M}$ and $5 \mu \mathrm{M}$ concentrations. The number of living cells was determined by cell counting 24 and 48 hours after drug treatment.

\section{Immunoblotting}

Immunoblotting was performed as described previously [31]. The following antibodies were used: H-Ras (Santa Cruz), SV40 Large-T (Santa Cruz), FLAG-Tag (Sigma), hTERT (Epitomics), Ku 70 (Cell Signaling), Ku 80 (Cell Signaling), $\alpha$-tubulin (Sigma) and $\beta$-actin (Abcam).

\section{Wound healing assay}

6-well plates were first coated with $10 \mu \mathrm{g} / \mathrm{ml}$ of collagen (Cohesion) for two hours, followed by blocking with BSA for one hour. $5 \times 10^{5}$ cells were seeded into the wells and allowed to grow until confluent. Following which, the cell monolayer was scratched at the bottom of the wells. Culture medium was then replaced with serum-free DMEM to minimize cell proliferation. Wells were observed under the microscope at different timepoints and the distance of gap was measured. The percentage of wound closure was calculated from distances quantified in three independent experiments.

\section{Boyden assay}

Migration assay was performed using cell culture inserts (BD Biosciences) with $8 \mu \mathrm{m}$ pore size. $10^{5}$ cells were diluted in $300 \mu \mathrm{l}$ of serum-free DMEM supplemented with $0.1 \%(\mathrm{w} / \mathrm{v}) \mathrm{BSA}$ and added to the upper chamber of the well. $500 \mu \mathrm{l}$ of normal culture DMEM supplemented with $100 \mathrm{ng} / \mathrm{ml}$ of human epidermal growth factor (ProSpec) was added to the lower chamber and incubated for 10 hours. Following which, medium was removed from both upper and lower chambers and cells were removed by swabbing from the upper chamber. Cells from the lower side of the insert were then stained with $1 \%$ crystal violet and observed under the microscope.

\section{Illumina microarray}

Total RNA was extracted using RNeasy Mini Kit (Qiagen). 500 ng total RNA was used for cRNA amplification using Illumina TotalPrep RNA Amplification kit (Ambion), following manufacturers' instructions. 750 ng cRNA was then hybridized onto the HumanRef-8 v2 Sentrix BeadChip (Illumina). Subsequently, the fluorescence emission by Cy3 was quantitatively detected by the Illumina BeadArray Reader software for downstream analysis of data by Partek software. Statistical significance of individual gene expression levels was analyzed by Analysis of variance (ANOVA) and significant genes were identified based on fold-changes. Threshold for significance was set at fold-change $> \pm|2.0|$ when compared to control cells. Absolute intensity differences between experimental groups were set at $p<0.05$. All data is MIAME compliant and the raw data has been deposited in a MIAME compliant database (accession number: GSE24097).

\section{RT-PCR and DNA gel electrophoresis}

cDNA was used in a total of $25 \mu \mathrm{L}$ PCR reactions containing PCR buffer, DNA Taq polymerase, $200 \mathrm{~nm}$ of each primer and dNTP. PCR products were resolved using gel electrophoresis.

\section{Metaphase spreads}

Metaphase spreads were prepared as described in Jeppesen's protocol [32], with slight modifications. Slides were observed using the Olympus Fluoview 1000 confocal microscopy system.

\section{G-band karyotyping and spectral karyotyping}

Cells at about $80 \%$ confluence were treated with colcemide for mitotic arrest and harvested by standard hypotonic treatment and methanol: acetic acid (3:1) fixation. For G-band karyotyping, slides were prepared by standard air drying method and G-band karyotype was performed according to the published protocols. For spectral karyotyping, slides were prepared by standard air drying method and hybridized with Human SKY paint probe (ASI), as per 
manufacturers' recommendations. 23 metaphases were analyzed by SKY analysis. Identified chromosomal abnormalities were described according to the International System for Human Cytogenetic Nomenclature (ISCN) (1995). Recurrent abnormalities are defined as at least 3 metaphase cells having the abnormality at the same region of chromosomal location or those that are involved in $>3 \%$ of cases.

\section{Coomassie blue staining and mass spectrometry}

Coomassie blue staining was performed on $7 \%$ and $15 \%$ SDS-PAGE gel. Bands were excised at $70 \mathrm{kDa}$ and the spots were rehydrated in digestion buffer containing sequencing grade modified trypsin at $37^{\circ} \mathrm{C}$. Digested peptides were extracted from gel with TFA extraction buffer and desalted using C-18 Zip-tips (Millipore). Mass spectra of the peptides in each sample were obtained by MALDI-TOF. Protein identification was based on peptide fingerprint mass mapping and peptide fragmentation mapping.

\section{Availability of supporting data}

The data sets supporting the results of this article are available in the LabArchves. The unique persistent identifier and hyperlink to data sets are listed below:

http://www.ncbi.nlm.nih.gov/geo/query/acc.cgi?

acc $=$ GSE24097 (Microarray data)

https://mynotebook.labarchives.com/share/Xu\%2520Cao/ MjAuOHw0MDkwNC8xNi9UcmV1Tm9kZS84MDAxM-

jY3Mjd8NTIuOA== (Additional file 1: Table S1)

https://mynotebook.labarchives.com/share/Xu\%2520

Cao/MjIuMXw0MDkwNC8xNy9UcmVlTm9kZS8zM-

jYONzI2ODU1fDU2LjE = (Additional file 2: Table S2)

https://mynotebook.labarchives.com/share/Xu\%

2520Cao/MjMuNHw0MDkwNC8xOC9UcmVlTm9kZS

80MTE5NjA3NzE4fDU5LjQ= (Additional file 3: Table S3)

\section{Additional files}

Additional file 1: Table S1. Summary of recurrent molecular

cytogenetic abnormalities detected in IMR90 RSH cells.

Additional file 2: Table S2. Ku70 is exclusively upregulated in IMR90-RSH cells but not IMR90 control cells.

Additional file 3: Table S3. Genes related to DNA damage response in IMR90 RSH and IMR90 hTERT cells when compared to IMR90 control cells.

\section{Abbreviations}

hTERT: Human telomerase reverse transcriptase; RSH: H-Ras, SV40 Large-T antigen and hTERT; DDR: DNA, Damage response; Dox: Doxorubicin; IL6: Interleukin-6;

IL8: Interleukin-8; COX-2: Cyclooxygenase-2; NHEJ: Non homologous end joining.

\section{Competing interests}

All authors declare that they have no competing interests.

\section{Author's contributions}

XC, CMK, KMM and XW designed assays, performed experiments, and analyzed data. YPL and VCR performed experiments and aided in data analysis. XC, CMK, and XW contributed to the writing and editing of the manuscript. All authors read and approved the final manuscript.

\section{Acknowledgements}

We sincerely thank Prof. Elizabeth Blackburn and Prof. Edison Liu for their contribution in conceiving the experiments. We thank Gerlynn Huang and Xiaojin Xie for technical assistance. We are extremely grateful to Dr. Tang Borluen and Tan May Chin for critical reading of the manuscript. This work was supported by Ministry of Education Academic Research Fund Tier 1 grants, R-183-000-320-112 to Xueying Wang, National University Health System (NUHS). Xu Cao and Chiou Mee Kong are recipients of research scholarships from Yong Loo Lin School of Medicine, NUS, NUHS, Singapore There is no conflict of interest that could be perceived to bias our work.

\section{Author details}

${ }^{1}$ Department of Biochemistry, Yong Loo Lin School of Medicine, National University of Singapore, Block MD4, Level 1, 5 Science Drive 2, Singapore 117545, Singapore. ${ }^{2}$ Gene Regulation Laboratory, Genome Institute of Singapore, Singapore, Singapore. ${ }^{3}$ National University Cancer Institute of Singapore (NCIS), Singapore, Singapore.

Received: 29 April 2014 Accepted: 29 July 2014

Published: 7 August 2014

\section{References}

1. Blackburn EH: Telomeres and telomerase: their mechanisms of action and the effects of altering their functions. FEBS Lett 2005, 579(4):859-862.

2. Greider CW: Chromosome first aid. Cell 1991, 67(4):645-647.

3. Watson JD: Origin of concatemeric T7 DNA. Nat New Biol 1972, 239(94):197-201.

4. Kim NW, Piatyszek MA, Prowse KR, Harley CB, West MD, Ho PL, Coviello GM, Wright WE, Weinrich SL, Shay JW: Specific association of human telomerase activity with immortal cells and cancer. Science 1994, 266(5193):2011-2015.

5. Janknecht R: On the road to immortality: hTERT upregulation in cancer cells. FEBS Lett 2004, 564(1-2):9-13.

6. Meyerson M, Counter CM, Eaton EN, Ellisen LW, Steiner P, Caddle SD, Ziaugra L, Beijersbergen RL, Davidoff MJ, Liu Q, Bacchetti S, Haber DA, Weinberg RA: hEST2, the putative human telomerase catalytic subunit gene, is up-regulated in tumor cells and during immortalization. Cell 1997, 90(4):785-795.

7. Newbold RF: The significance of telomerase activation and cellular immortalization in human cancer. Mutagenesis 2002, 17(6):539-550.

8. Hanahan D, Weinberg RA: Hallmarks of cancer: the next generation Cell 2011, 144(5):646-674.

9. Li S, Rosenberg JE, Donjacour AA, Botchkina IL, Hom YK, Cunha GR, Blackburn EH: Rapid inhibition of cancer cell growth induced by lentiviral delivery and expression of mutant-template telomerase RNA and antitelomerase short-interfering RNA. Cancer Res 2004, 64(14):4833-4840.

10. Chang S, Khoo CM, Naylor ML, Maser RS, DePinho RA: Telomere-based crisis: functional differences between telomerase activation and ALT in tumor progression. Genes Dev 2003, 17(1):88-100.

11. Stewart SA, Hahn WC, O'Connor BF, Banner EN, Lundberg AS, Modha P, Mizuno $H$, Brooks MW, Fleming M, Zimonjic DB, Popescu NC, Weinberg RA: Telomerase contributes to tumorigenesis by a telomere length-independent mechanism. Proc Natl Acad Sci U S A 2002, 99(20):12606-12611.

12. Bryan TM, Reddel RR: Telomere dynamics and telomerase activity in in vitro immortalised human cells. Eur J Cancer 1997, 33(5):767-773.

13. Hahn WC, Counter CM, Lundberg AS, Beijersbergen RL, Brooks MW, Weinberg RA: Creation of human tumour cells with defined genetic elements. Nature 1999, 400(6743):464-468.

14. Durkin JP, Chakravarthy B, Mealing G, Schwartz JL, Tremblay R, Whitfield JF, Franks DJ: The role of signal-transducing events in the proliferative response of cells to a mitogenic viral K-ras protein. Cell Signal 1990, 2(3):285-297.

15. Borchert S, Czech-Sioli M, Neumann F, Schmidt C, Wimmer P, Dobner T, Grundhoff A, Fischer N: High-affinity Rb binding, p53 inhibition, subcellular localization, and transformation by wild-type or tumor-derived shortened Merkel cell polyomavirus large T antigens. J Virol 2014, 88(6):3144-3160.

16. Duan HW, Bin P, Liu QJ, Wang YD, Niu Y, Liu Q, Dai YF, Chen W, Zheng YX: [Cytotoxicity and genomic damage of benzo[a]pyrene in gene 
transformed cell model]. Zhonghua Yu Fang Yi Xue Za Zhi 2010, 44(4):314-318.

17. Mason DX, Keppler D, Zhang J, Jackson TJ, Seger YR, Matsui S, Abreo F, Cowell JK, Hannon GJ, Lowe SW, Lin AW: Defined genetic events associated with the spontaneous in vitro transformation of ElA/Rasexpressing human IMR90 fibroblasts. Carcinogenesis 2006, 27(2):350-359.

18. Hanahan D, Weinberg RA: The hallmarks of cancer. Cell 2000, 100(1):57-70.

19. Lowe SW, Ruley HE, Jacks T, Housman DE: p53-dependent apoptosis modulates the cytotoxicity of anticancer agents. Cell 1993, 74(6):957-967.

20. Ciapponi L, Cenci G, Ducau J, Flores C, Johnson-Schlitz D, Gorski MM, Engels WR, Gatti M: The Drosophila Mre11/Rad50 complex is required to prevent both telomeric fusion and chromosome breakage. Curr Biol 2004, 14(15):1360-1366

21. Chiang AC, Massague J: Molecular basis of metastasis. N Engl J Med 2008, 359(26):2814-2823.

22. Fidler IJ, Kripke ML: Metastasis results from preexisting variant cells within a malignant tumor. Science 1977, 197(4306):893-895.

23. Imamura H, Matsuyama Y, Tanaka E, Ohkubo T, Hasegawa K, Miyagawa S, Sugawara Y, Minagawa M, Takayama T, Kawasaki S, Makuuchi M: Risk factors contributing to early and late phase intrahepatic recurrence of hepatocellular carcinoma after hepatectomy. J Hepatol 2003, 38(2):200-207.

24. Ghosh A, Saginc G, Leow SC, Khattar E, Shin EM, Yan TD, Wong M, Zhang Z, Li G, Sung WK, Zhou J, Chng WJ, Li S, Liu E, Tergaonkar V: Telomerase directly regulates NF-kappaB-dependent transcription. Nat Cell Biol 2012, 14(12):1270-1281.

25. Jovanovic M, Vicovac L: Interleukin-6 stimulates cell migration, invasion and integrin expression in HTR-8/SVneo cell line. Placenta 2009, 30(4):320-328.

26. Liu Z, Li Q, Li K, Chen L, Li W, Hou M, Liu T, Yang J, Lindvall C, Bjorkholm M, Jia J, Xu D: Telomerase reverse transcriptase promotes epithelial-mesenchymal transition and stem cell-like traits in cancer cells. Oncogene 2013, 32(36):4203-4213.

27. Menter DG, Dubois RN: Prostaglandins in cancer cell adhesion, migration, and invasion. Int J Cell Biol 2012, 2012:723419.

28. Yang WH, Lan HY, Huang CH, Tai SK, Tzeng CH, Kao SY, Wu KJ, Hung MC, Yang MH: RAC1 activation mediates Twist1-induced cancer cell migration. Nat Cell Biol 2012, 14(4):366-374.

29. Walker JR, Corpina RA, Goldberg J: Structure of the Ku heterodimer bound to DNA and its implications for double-strand break repair. Nature 2001, 412(6847):607-614.

30. Tamura K, Adachi Y, Chiba K, Oguchi K, Takahashi H: Identification of Ku70 and Ku80 homologues in Arabidopsis thaliana: evidence for a role in the repair of DNA double-strand breaks. Plant J 2002, 29(6):771-781.

31. Zhang $Y$, Toh $L$, Lau $P$, Wang $X$ : Human telomerase reverse transcriptase (hTERT) is a novel target of the Wnt/beta-catenin pathway in human cancer. J Biol Chem 2012, 287(39):32494-32511.

32. Jeppesen P: Immunofluorescence in cytogenetic analysis: method and applications. Genet Mol Biol 2000, 23(4):1003-1014.

doi:10.1186/1471-2091-15-17

Cite this article as: Cao et al.: The use of transformed IMR90 cell model to identify the potential extra-telomeric effects of $h T E R T$ in cell migration and DNA damage response. BMC Biochemistry 2014 15:17.

\section{Submit your next manuscript to BioMed Central and take full advantage of:}

- Convenient online submission

- Thorough peer review

- No space constraints or color figure charges

- Immediate publication on acceptance

- Inclusion in PubMed, CAS, Scopus and Google Scholar

- Research which is freely available for redistribution

Submit your manuscript at www.biomedcentral.com/submit 